\title{
Laryngeal Dysplasia: What does the Evidence Tell Us?
}

\author{
${ }^{1}$ Anusha Balasubramanian, ${ }^{2}$ Vinidh Paleri
}

\begin{abstract}
Management of laryngeal dysplasia often poses a clinical conundrum, especially so with its unpredictable propensity for malignant transformation. The wide heterogeneity in published results and the dearth of level I evidence makes it challenging to arrive at a consensus or best practice guidelines. In 2010, ENT UK had developed such a guideline based on critical analysis of previously published data and professional opinion. This article examines the available evidence, and attempts to highlight the best possible modalities in investigation and management strategies as objectively as possible.
\end{abstract}

Keywords: Carcinoma-in Situ, Dysplasia, Laryngeal, Leukoplakia, Vocal fold.

How to cite this article: Balasubramanian A, Paleri V. Laryngeal Dysplasia: What does the Evidence Tell Us? International Journal of Head and Neck Surgery, 2018;9(2):87-93.

\section{Source of support: Nil}

Conflict of interest: None

\section{INTRODUCTION}

Dysplasia is a histological diagnosis in the presence of abnormal maturation of cells and disorderly growth from the base upward. Confirmation of the diagnosis on histopathological examination would require the following cytologic characteristics: hyperchromatism, increased nuclear-cytoplasmic ratio, poikilocytosis and anisocytosis, coupled with architectural changes which include but not limited to basal cell hyperplasia, abnormal mitoses, and drop-shaped rete ridges. ${ }^{1}$ It is these features that form the foundation for accurate histological interpretation, regardless of the classification used.

Atypia refers to individual cell changes, whereas dysplasia refers to the changes in the epithelium as a whole. ${ }^{2}$ These changes can be appreciated under light microscopy, and the degree of nuclear atypia determines

\footnotetext{
${ }^{1}$ Otorhinolaryngologist-Head and Neck Surgeon, ${ }^{2}$ Professor

${ }^{1}$ Department of Otorhinolaryngology-Head and Neck Surgery, Kuala Lumpur Hospital, Jalan Pahang, Kuala Lumpur, Malaysia

${ }^{2}$ Department of Head and Neck Surgery, The Royal Marsden National Health Service Foundation Trust, London, UK

Corresponding Author: Vinidh Paleri, Professor, Department of Head and Neck Surgery, The Royal Marsden National Health Service Foundation Trust, London, UK, e-mail: vinidh. paleri@rmh.nhs.uk
}

the grade severity. ${ }^{3}$ Regardless of the grade severity, the basement membrane of the cell remains intact; once the basement membrane is breached, it is then termed as invasive carcinoma and no longer precancerous lesion.

Repeated injury to squamous epithelium from carcinogenic substances, tobacco smoke for instance, would result in hyperplastic followed by dysplastic changes arising from the cell's inbuilt mechanism of coping with insult and repair. The vocal folds are lined by nonkeratinized stratified squamous epithelium, similar to the oral cavity mucosa in the propensity to develop premalignant lesions, and the subsequent potential for malignant transformation.

Laryngeal dysplasia has been reported to occur in 2-10 per 100,000 population, ${ }^{4}$ with a pooled overall malignant transformation rate (MTR) of $14 \%$ (CI 8-22\%). ${ }^{5}$ Although a higher grade lesion would mean a higher risk of malignant transformation, ${ }^{5}$ in which mild/moderate lesions have a $10.6 \%$ risk and severe/carcinoma in situ (CIS) have a threefold higher $(30.4 \%$ ) risk of transforming, the time to progression is not dependent on the severity. ${ }^{5}$ The mean time to malignant transformation is 5.8 years, ${ }^{5}$ hence the emphasis on the need for judicious follow-up. It is not surprising to see a mild dysplasia progressing directly to invasive carcinoma or a severe dysplasia remaining indolent. Clinical progression of laryngeal dysplasias from mild/moderate/severe dysplasia to CIS, and subsequent invasive carcinoma is not often observed, possibly owing to the natural history of the disease is such, sampling errors and variability in pathology reporting, ${ }^{6}$ in turn giving rise to the wide variation in management strategies.

\section{HISTOLOGICAL GRADING}

A histological grading system requires consistency in interpretation, reproducibility, clinical applicability and the ability to predict risk of malignant transformation. Numerous grading systems have been reported in the past for laryngeal intraepithelial lesions. The WHO 2005 classification espoused the three-tier system of mild, moderate and severe dysplasia. However, several interobserver studies, ${ }^{7.8}$ in which pathologists used all three classifications, showed no significant advantage with only moderate inter-observer agreements. The 4th Edition of the World Health Organization Classification of Head and Neck Tumours, published in $2017,{ }^{9}$ has adopted a 
two-tier system, based on the morphological criteria of the amended Ljubljana classification, where the lesions are called squamous intraepithelial lesions (SIL): low-grade SILs and high-grade SILs. ${ }^{10}$

\section{CLINICAL ASSESSMENT}

Patients with laryngeal dysplasia may be asymptomatic or present with hoarseness, and a leukoplakia or erythroplakia patch may be evident on flexible nasendoscopy in the clinic setting. The vibratory properties of the superficial lamina propria (SLP) is affected by this hyperkeratotic patch and may be demonstrable on videostroboscopy, should this facility be available, although not mandatory. Photo-documentation of the lesion at presentation is highly recommended as a baseline reference, as well as for comparison purposes during follow-up. ${ }^{11}$

Although individual studies have demonstrated the benefits of narrow band imaging (NBI) in head and neck cancerous and pre-cancerous lesions, a recent systematic review failed to pool data on such efficacy in laryngeal precancerous lesions due to the wide heterogeneity in published results. ${ }^{12}$ Nevertheless, high diagnostic accuracy rates have been reported. In a prospective study of 158 patients, DeVito et al. ${ }^{13}$ reported a high sensitivity rate of $97 \%$ (CI, 84.2-99.9\%), and specificity rate of $92.5 \%$ (CI, 79.6-98.4\%) in identifying early laryngeal cancer and precancerous lesions. Kraft et al. ${ }^{14}$ showed high sensitivity rate of $97 \%$ for NBI (versus $79 \%$ for white light assessment), in 205 patients who underwent microlaryngoscopy, in picking up laryngeal cancer and precursor lesions; the specificity rates were similar for NBI and whitel light at 95 and 96\%, respectively. Clinicians should consider NBI use where available, thus minimizing inter observer variability arising from subjective clinical assessment with pure white light imaging alone.

\section{TREATMENT STRATEGIES}

The decision on treatment modality is a shared process between the treating surgeon and the patient, and should ideally consider the patient's comorbidities, preferences, fitness for intervention, histological grading, extent of lesion and availability of treatment facility. The patient must be advised of the anticipated changes in voice quality due to vocal fold scarring, more so for revision procedures. At present, the consensus statement developed by ENT UK in 2010 is probably the best available practice guidelines to fall back on as a reference point. A flowchart developed by Cosway and Paleri utilizing the contents of the consensus statement elegantly summarizes and describes the stepwise approach to management of patients with laryngeal dysplasia (Flow chart 1). ${ }^{15}$

Management is essentially guided by the appearance of the lesion i.e., single/multiple foci versus widespread disease, and may be summarized as follows: ${ }^{11}$

Single/multiple lesion must be completely excised in the initial seating up to all visible margins as possible, as the primary excision has a dual role of diagnostic and therapeutic purpose. On the other hand, histologic mapping with multiple biopsies is advocated for widespread confluent lesions, and staged resection to be performed in subsequent seating. Cold steel instrument or $\mathrm{CO}_{2}$ laser is the recommended surgical tool. Vocal cord stripping is not recommended, contrary to that practiced in Ljubljana, where in all patients with atypical hyperplasia were subjected to this procedure.

The importance of specimen presentation to obtain accurate results cannot be emphasized enough. As part of the recommendation, all specimens must be mounted, orientated and presented on an anatomic template for photo-documentation and histologic interpretation. Larger biopsies are preferable when possible, to enable more accurate orientation and interpretation of the lesion. Presence of dysplasia at surgical margins is not an indication for further biopsy or excision. However, the patient must be followed through and a low-threshold for rebiopsy should be the practice in the event of a new lesion or change in appearance of preexisting lesion. The management of patients with atypical hyperplasia/ severe dysplasia and CIS should ideally be discussed at a multidisciplinary meeting.

Although the rate of MTR has been documented to be lower in patients undergoing surgical excision 15\% (CI, 12-18\%) compared to patients in the 'non-excision' group $21 \%$ (CI, 16-27\%) this result could not reach statistical significance $(p=0.12)$ despite adjustment for grade, and therefore a precise distinction and superiority of either treatment modality has not be made thus far. ${ }^{5}$

The concordance of NBI endoscopy findings with histopathological results of vocal fold leukoplakic lesions has been proven to be statistically significant with a kappa index of $0.77,(p<0.001) .{ }^{16}$ The use of NBI is advocated when available for its superior quality in highlighting neovascularization and the ability to delineate more precise margins of a vocal fold lesion, especially in the follow-up setting.

\section{FOLLOW-UP RECOMMENDATION}

Laryngeal dysplasia is classified into 2 categories, i.e high risk and low risk lesions for the purpose of follow-up as per the consensus statement. ${ }^{11,15}$ The low risk category consists of mild/moderate dysplasia 
Flow chart $1 \mathrm{~A}$ and B: Algorithm showing management options for laryngeal dysplasia reproduced with permission from Cosway and Paleri ${ }^{15}$

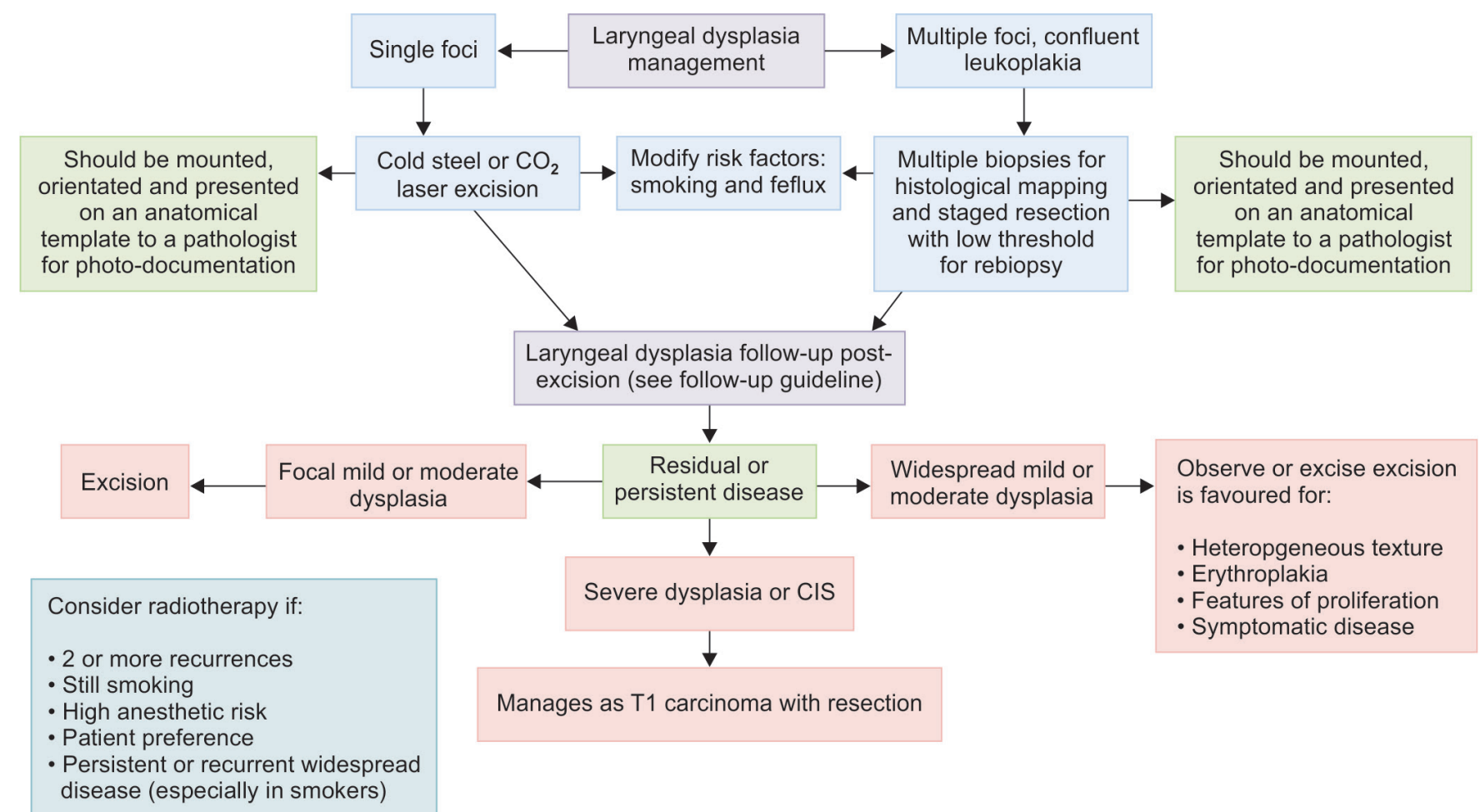

A

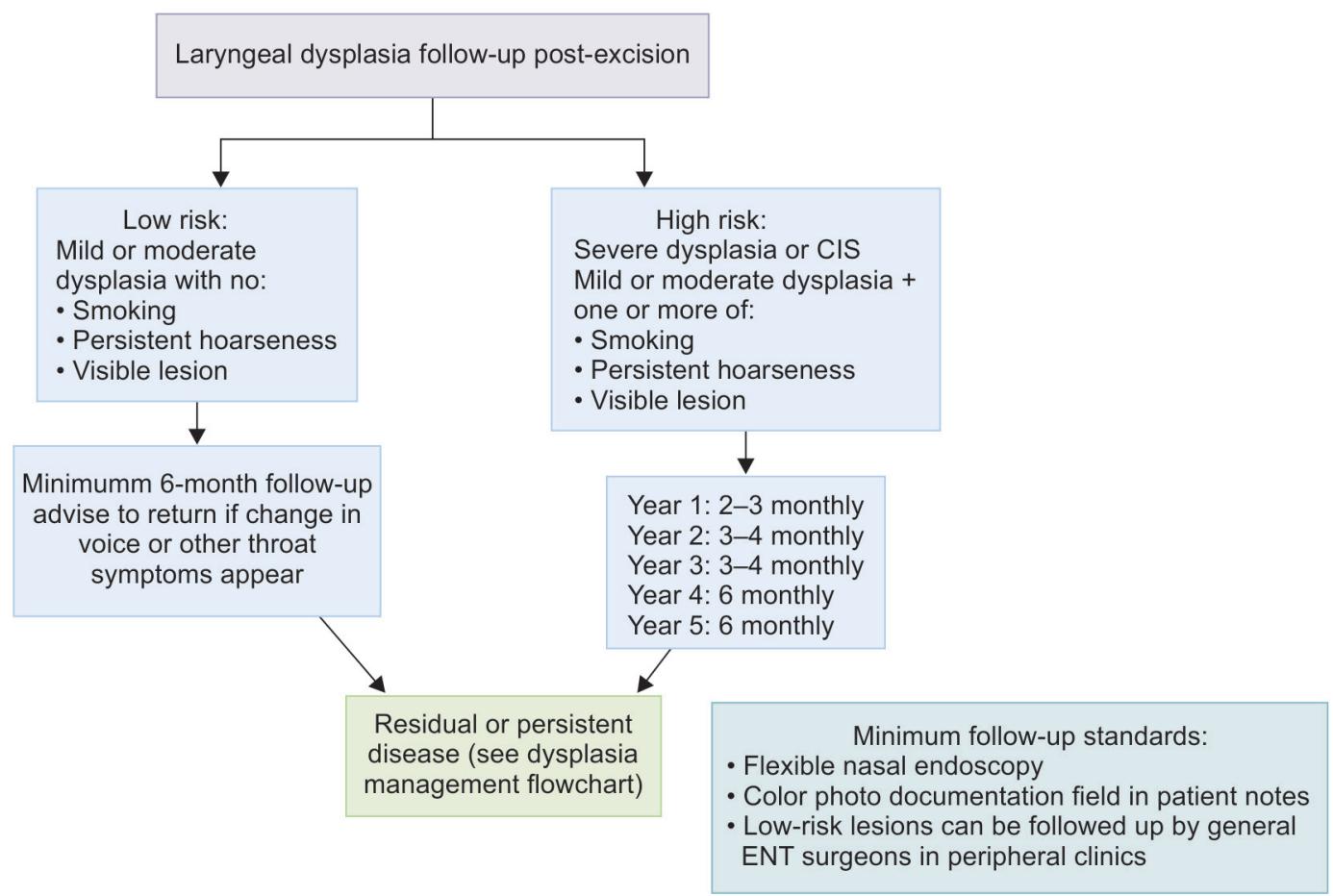

in the absence of smoking, persistent hoarseness or visible lesion. These patients must be followed-up for a minimum period of 6 months and advised to return in the event of new 'throat' symptoms or change in voice. Severe dysplasia/CIS as per WHO grading or, mild/ moderate dysplasia with presence of either smoking, persistent hoarseness or visible lesion are categorized as high risk. It is this group that must be followed up as per T1 laryngeal carcinoma protocol.

Flexible nasendoscopy and colour photo-documentation are the basic requirements during each visit. A recent secondary analysis of preexisting data by Paleri et al. has highlighted the benefits and role of NBI on follow-up of patients with laryngeal dysplasia. ${ }^{17}$ The treating physician is 4 times less likely to miss a malignant transformation with the use of NBI during follow-up in the case of severe dysplasia. Hence, a negative finding on NBI would allow monitoring the patient in an outpatient setup, and 
negate the necessity for unnecessary repeated biopsies, and subsequent worsening of voice quality. ${ }^{17}$

Although full thickness biopsies are essential for diagnosis, a recent longitudinal retrospective analysis demonstrated that repeated full-thickness excisions / biopsies do not confer therapeutic regression of dysplasia in terms of reversal of progression or downgrading of severity, ${ }^{6}$ contrary to that previously reported. ${ }^{18}$ In fact, the odds of dysplasia worsening had increased by $4 \%$ with each additional excision. ${ }^{6}$ The aforementioned findings reinforce the use of NBI in surveillance and surgery, to cautiously avoid over-zealous treatment.

\section{MANAGEMENT OF RECURRENT/PERSISTENT DISEASE}

Severe dysplasia / CIS must be managed as per T1 laryngeal carcinoma. Management of mild/moderate dysplastic lesions again depends on whether they are focal or widespread. Focal lesions should be excised where possible, whereas cohesive lesions may be excised or observed. Excision in this case is recommended when patients are symptomatic or there is evidence of change in the appearance of the lesion. ${ }^{11,15}$

Radiotherapy may be considered in active smokers, patients with 2 or more recurrences, patients with high anaesthetic risks, widespread persistent/recurrent disease especially so in smokers or if radiotherapy is the patient's modality of choice. ${ }^{11,15}$

\section{SURGICAL PROCEDURES AND LASERS}

Serial microflap excision has been the practice norm and believed to have significant benefits with regard to oncologic outcome, ${ }^{18}$ till recently challenged by a longitudinal study. ${ }^{6}$ Serial excisions however result in poorer voice quality. A recent single-stage excision method of combining submucosal infusion technique with microflap excision described by Kono et al., has demonstrated safe oncologic clearance with satisfactory vocal function; ${ }^{19}$ however, this would require further research.

Besides providing increased precision and an unobstructed view of the surgical field compared to microflap surgery, lasers minimize tissue manipulation, intraoperative bleeding and surrounding tissue damage. ${ }^{20}$ Carbon dioxide $\left(\mathrm{CO}_{2}\right)$ laser has long been the workhorse of laryngeal surgery in general, and for laryngeal dysplasia specifically. In recent times, pulsed dye laser (PDL) and potassium titanyl phosphate (KTP) lasers have gained their respective roles in laryngeal surgery. They not only photoangiolyse the microvasculature of the superficial lamina propria (SLP), but affect the extracellular matrix and connective tissue, and therefore are able to target vascular lesions while preserving the surface epithelium. ${ }^{21}$ The characteristics of various lasers as described by Yan et al. ${ }^{21}$ are summarized in Table 1.

The preferential use of PDL over KTP in laryngeal dysplasia might be guided by the fact that PDL does not only create a cleavage plane between the basement membrane and SLP, but penetrates deeper than KTP, conferring an advantage in thick lesions. ${ }^{21,22}$ Lesions in the non-phonatory region of the vocal fold are better off treated with PDL as the basement membrane and SLP remain unaffected. ${ }^{21}$

While serial in-office use of lasers for leukoplakic lesions might control disease effectively, minimize

Table 1: Types of lasers utilized in laryngeal surgery and their characteristics

\begin{tabular}{llll}
\hline Laser type/Characteristics & $\mathrm{CO}_{2}$ & KTP & $P D L$ \\
\hline Rigid & Yes & No & No \\
Fiber-optic transmission & Yes (flexible type) & Yes & Yes \\
Office use (LA) & Yes (flexible type only) & Yes & Yes \\
Operating theater use (GA) & Yes & Yes & Yes \\
Wavelength (nm) & 10,600 & 532 & 585 \\
Target chromophore & Water & Hemoglobin & Hemoglobin \\
Mechanism & Cutting/ablating & Photoangiolytic & Photoangiolytic \\
Specific characteristics & Continuos mode preferred for & Allows for contact/ & Allows for contact/non-contact \\
& coagulative property & non-contact mode & mode \\
& Pulsed mode preferred for & Better hemostatic effect & Better hemostatic effect than \\
& incisions & than CO & CO \\
& Minimal surrounding tissue & Wide pulse width & Short pulse width \\
& damage & & Risk of vessel wall rupture prior \\
& More focused than KTP/PDL & Slower heating & to completion of hemostasis \\
\hline
\end{tabular}


morbidity and preserve voice quality, ${ }^{23}$ simultaneously reducing cost from operating theatre charges and hospitalization fees, judicious patient selection is crucial in minimizing complication rates and repeated procedures. ${ }^{24}$ Earlier operative intervention might benefit patients who require repeated six-monthly in-office procedures. ${ }^{23}$

Nevertheless, selection of one laser type over the other ultimately boils down to the principle of balancing thermal damage and its efficacy on target tissue, availability of the equipment and trained personnel and preference of the surgeon.

\section{BIOMARKERS}

Biomarkers are genes or proteins detected in precancerous or malignant lesion and have a crucial role in predicting malignant transformation. Although the scoring systems for biomarker staining vary widely among studies, presence of cortactin, cyclin D1 and Ki67 from individual studies have demonstrated statistically significant progression to malignancy when these biomarkers were positive. ${ }^{25-27}$ The wide heterogeneity in scoring systems and published results of these biomarkers have made attempts at meta-analysis not possible or the results of such attempts insignificant.

The presence of p53 on the other hand is inversely related to malignant transformation. The results of a pooled meta-analysis on $\mathrm{p} 53$ proved to be statistically insignificant in predicting malignant transformation. ${ }^{28}$ In conclusion, there is insufficient evidence on the role of biomarkers to predict malignant transformation of laryngeal precancerous lesions at present. ${ }^{28}$

\section{RISK FACTOR REDUCTION AND CHEMOPREVENTION}

The detection of human papilloma virus (HPV) load depends on the processing method, i.e., polymerase chain reaction, in situ hybridization or immunohistochemistry staining. ${ }^{29}$ Several small cohorts have failed to demonstrate a positive association between HPV with laryngeal premalignancy. ${ }^{30-32}$ There is a need for substantial high quality evidence to demonstrate either a causal effect or negative relationship between HPV and laryngeal dysplasia.

The carcinogenic properties of alcohol, although not fully understood, is thought to arise from the effect exerted by acetylaldehyde; a metabolite of alcohol, onto epithelial cells or due to direct contact of alcohol with surface epithelieum. ${ }^{33}$ A recent meta-analysis by Bagnardi et al. demonstrated a pooled relative risk of 2.65 of developing laryngeal cancers in heavy drinkers as compared to occasional and non-drinkers; with a positive dose-risk relationship. ${ }^{34}$
A small retrospective analysis has described that continuation or cessation of smoking does not influence the progression of laryngeal dysplasia to malignancy. ${ }^{35}$ This perhaps could be attributed to the irreversible genetic and morphologic changes incurred by smoking. ${ }^{2}$ Although tobacco smoking is an established risk factor for laryngeal cancers and produces a synergistic effect with alcohol in carcinoma formation, current knowledge as demonstrated in a meta-analysis by Weller et al. dictates that there is insufficient evidence to conclude on the harmful effects of smoking or alcohol on malignant transformation of laryngeal dysplastic lesions. ${ }^{5}$

Beside the free radical action and direct insult on laryngeal mucosa, hydrochloropeptic complex, the main active component of gastric acid secretion, exerts it damaging effect at the junctional intercellular structures, resulting in increased permeability, raised intercellular acidity, osmotic disruption and subsequent cell necrosis. ${ }^{36}$ Inactivated trypsin, a component of duodenalgastric reflux, exerts its proteolytic effect by breaking down cell connection. A statistically significant association is noted in reflux being a risk factor for precancerous and squamous malignancies of the laryngo-pharyngeal complex; especially so with prolonged exposure of over 20 years. ${ }^{36}$ A direct causal-effect relationship of acid reflux and formation of cancer has yet to be demonstrated probably due to the simultaneous presence of smoking and alcohol consumption as confounding risk factors in studied patients.

It is nevertheless sensible to advocate cessation of smoking and drinking, as well as place patients on anti-reflux therapy, as recommended in the consensus statement. ${ }^{11}$ Enrolment into smoking cessation programs should be offered to patients who face difficulty in dropping this habit.

The natural course of normal cells evolving to cancerous cells is determined by the effect of field cancerization and multistep carcinogenesis. ${ }^{37}$ These in turn are influenced by genetic instability (loss of heterozygosity in $9 p$ and $3 p$ ) and environmental exposure of carcinogens. ${ }^{37,38}$ These neoplastic principles form the fundamentals for chemoprevention intervention and development of targeted therapies.

Folate deficiency causes genetic instability by breakage of DNA as a result of assimilation of uracil within DNA, and in turn promoting carcinogenesis. ${ }^{39}$ Primary chemoprevention with oral folic acid supplement might be beneficial in halting the progression of laryngeal precancerous lesion, ${ }^{39}$ but further controlled trials are required to establish its role. At present, smoking cessation is the best intervention to prevent recurrences. The limited number of chemoprevention trials in laryngeal dysplasia might be attributed to the limitations in 
accessibility and monitoring of lesion within the larynx; in contrast to oral precancerous lesion, that are conveniently accessed and well researched.

\section{LIMITATIONS}

Meta-analyses and systematic reviews of either management strategies or biomarkers were mostly of level 3 and 4 evidence consisting of retrospective studies and case series, except for one prospective study in Weller's systematic review ${ }^{5}$ and the various prospective studies on NBI. Nevertheless, the pooled MTR and time to malignant transformation were statistically significant. Further well planned prospective trials and future research into the role of biomarkers and genetic analysis would shed light on new pertinent information in formulation of future guidelines to direct more appropriate management of laryngeal dysplasia. Comparative studies on the efficacy of various treatment modalities, influence of smoking and alcohol consumption on malignant transformation, and role of chemoprevention is a scope to consider as well.

\section{CONCLUSION}

The challenge in the management of laryngeal dysplasia remains in finding the balance between achieving oncologic clearance and acceptable functional outcome. The natural course of the disease is nonlinear. Severe dysplasia and CIS demonstrate a higher rate of malignant transformation. Grade of dysplasia still dictates treatment decision and every effort possible should be made to reduce observer variability. Narrow band imaging has an established role in follow-up of patients. Smoking cessation must be advocated to prevent recurrence.

\section{REFERENCES}

1. Warnakulasuriya S, Reibel J, Bouquot J, Dabelsteen E. Oral epithelial dysplasia classification systems: predictive value, utility, weaknesses and scope for improvement. J Oral Pathol Med 2008 Mar;37(3):127-133.

2. Ferlito A, Devaney KO, Woolgar JA, Slootweg PJ, Paleri V, Takes RP, Strojan P, Bradley PJ, Rinaldo A. Squamous epithelial changes of the larynx: diagnosis and therapy. Head Neck 2012 Dec;34(12):1810-1816.

3. Barnes L, Eveson JW, Reichart P, Sidransky D. World Health Organization Classification of Tumours. Pathology and Genetics of Tumours of the Head and Neck. IARC Press, Lyon; ed 2005.

4. Bouquot J, Gnepp D. Laryngeal precancer: a review of the literature, commentary, and comparison with oral leukoplakia. Head Neck 1991;13:488-497.

5. Weller MD, Nankivell PC, McConkey C, Paleri V, Mehanna $\mathrm{HM}$. The risk and interval to malignancy of patients with laryngeal dysplasia; a systematic review of case series and metaanalysis. Clin Otolaryngol 2010 Oct;35(5):364-372.

6. Ahn A, Wang L, Slaughter JC, Nguyen AM, Ossoff RH, Francis DO. Serial Full-Thickness Excision of Dysplastic
Vocal Fold Leukoplakia: Diagnostic or Therapeutic?. Laryngoscope 2016 Apr;126(4):923-927.

7. McLaren KM, Burnett RA, Goodlad JR, Howatson SR, Lang $\mathrm{S}$, Lee FD, et al. Consistency of histopathological reporting of laryngeal dysplasia. The Scottish pathology consistency group. Histopathology. 2000;37(5):460-463.

8. Fleskens SA, Bergshoeff VE, Voogd AC, van Velthuysen ML, Bot FJ, Speel EJ, et al. Interobserver variability of laryngeal mucosal premalignant lesions: a histopathological evaluation. Mod Pathol. 2011;24(7):892-898.

9. Gale N, Poljak M, Zidar N. Update from the 4th Edition of the World Health Organization Classification of Head and Neck Tumours: What is New in the 2017 WHO Blue Book for Tumours of the Hypopharynx, Larynx, Trachea and Parapharyngeal Space. Head Neck Pathol. 2017 Mar;11(1):23-32.

10. Gale N, Gnepp DR, Poljak M, Strojan P, Cardesa A, Helliwell T, et al. Laryngeal squamous intraepithelial lesions: an updated review on etiology, classification, molecular changes, and treatment. Adv Anat Pathol. 2016;23(2):84-91.

11. Mehanna H, Paleri V, Robson A, Wight R, Helliwell T. Consensus statement by otorhinolaryngologists and pathologists on the diagnosis and management of laryngeal dysplasia. Clin Otolaryngol 2010 Jun;35(3):170-176.

12. Cosway B, Drinnan M, Paleri V. Narrow band imaging for the diagnosis of head and neck squamous cell carcinoma: A systematic review. Head Neck 2016 Apr;38 Suppl 1:E2358-2367.

13. De Vito A, Meccariello G, Vicini C. Narrow band imaging as screening test for early detection of laryngeal cancer: a prospective study. Clin Otolaryngol 2017Apr;42(2):347-353.

14. Kraft M, Fostiropoulos K, Gürtler N, Arnoux A, Davaris N, Arens $\mathrm{C}$. Value of narrow band imaging in the early diagnosis of laryngeal cancer. Head Neck 2016Jan;38(1):15-20.

15. Cosway B, Paleri V. Laryngeal dysplasia: an evidence-based flowchart to guide management and follow up. J Laryngol Otol 2015 Jun;129(6):598-599.

16. Staníková L, Šatanková J, Kučová H, Walderová R, Zeleník $\mathrm{K}$, Komínek P. The role of narrow-band imaging (NBI) endoscopy in optical biopsy of vocal cord leukoplakia. Eur Arch Otorhinolaryngol 2017Jan;274(1):355-359.

17. Paleri V, Sawant R, Mehanna H, Ainsworth H, Stocken D. Laryngeal Dysplasia and narrow band imaging: secondary analysis of published data supports the role in patient follow up. Clin Otolaryngol (in press)

18. Schweinfurth JM, Powitzky E, Ossoff RH. Regression of laryngeal dysplasia after serial microflap excision. Ann Otol Rhinol Laryngol 2001;110:811-814.

19. Kono T, Saito K, Yabe H, Ogawa K. Phonosurgical resection using submucosal infusion technique for precancerous laryngeal leukoplakia. Laryngoscope 2017 Jan;127(1):153-158.

20. Koufman JA, Rees CJ, Frazier WD, Kilpatrick LA, Wright SC, Halum SL, et al. Office-based laryngeal laser surgery: a review of 443 cases using three wavelengths. Otolaryngol Head Neck Surg 2007Jul;137(1):146-151.

21. Yan Y, Olszewski AE, Hoffman MR, Zhuang P, Ford CN, Dailey SH, et al. Use of Lasers in Laryngeal Surgery. J Voice 2010 Jan;24(1):102-109.

22. Franco JRA Jr. In-office laryngeal surgery with the 585-nm pulsed dye laser. Curr Opin Otolaryngol Head Neck Surg 2007Dec;15(6):387-393.

23. Koss SL, Baxter P, Panossian H, Woo P, Pitman MJ. Serial inoffice laser treatment of vocal fold leukoplakia: Disease control and voice outcomes. Laryngoscope 2017Jul;127(7):1644-1651. 
24. Del Signore AG, Shah RN, Gupta N, Altman KW, Woo P. Complications and Failures of Office-Based Endoscopic Angiolytic Laser Surgery Treatment. J Voice 2016Nov;30(6):744-750.

25. Rodrigo JP, García-Carracedo D, García LA, Menéndez S, Allonca E, González MV, et al. Distinctive clinicopathological associations of amplification of the cortactin gene at 11 13 in head and neck squamous cell carcinomas. J Pathol 2009Mar;217(4):516-523.

26. Papadimitrakopoulou V, Izzo JG, Liu DD, Myers J, Ceron TL, Lewin J, et al. Cyclin D1 and cancer development in laryngeal premalignancy patients. Cancer Prev Res 2009Jan;2(1):14-21.

27. Mirza S, Jeannon JP, Soames J, Wilson JA. Is Ki67 a marker for the transformation of laryngeal dysplasia to carcinoma?. Acta Oto-Laryngol 2006Apr;126(4):418-422.

28. Nankivell P, Weller M, McConkey C, Paleri V, Mehanna H. Biomarkers in laryngeal dysplasia:a systematic review. Head Neck 2011Aug;33(8):1170-1176.

29. Nowińska K, Ciesielska U, Podhorska-Okołów M, Dzięgiel P. The role of human papillomavirus in oncogenic transformation and its contribution to the etiology of precancerous lesions and cancer of the larynx: A review. Adv Clin Exp Med 2017 May-Jun;26(3):539-547.

30. Gallo, A, Degener, AM, Pagliuca, G. Detection of human papillomavirus and adenovirus in benign and malignant lesions of the larynx. Otolaryngol Head Neck Surg. 2009 Aug;141(2): 276-281.

31. Waters HH, Seth R, Hoschar AP, Benninger MS. Does HPV have a presence in diffuse high grade pre-malignant lesions of the larynx? Laryngoscope 2010;120 Suppl 4:S201.
32. Pagliuca G, Martellucci S, Degener AM, Pierangeli A, Greco A, Fusconi M, et al. Role of Human Papillomavirus in the Pathogenesis of Laryngeal Dysplasia. Otolaryngol Head Neck Surg 2014 Jun;150(6):1018-1023.

33. Boffetta P, Hashibe M. Alcohol and cancer. Lancet Oncol 2006 Feb;7(2):149-156.

34. Bagnardi V, Rota M, Botteri E, Tramacere I, Islami F, Fedirko $\mathrm{V}$, et al. Alcohol consumption and site-specific cancer risk: a comprehensive dose-response meta-analysis. Br J Cancer 2015Feb 3;112(3):580-593.

35. Theodosiou MG, Yiotakis J, Dikoglou C, Lazaris AC, Athanasiadis-Sismanis A, Xenellis J. Laryngeal dysplasia: a long-term follow-up study. J BUON 2013Jul-Sep;18(3):683-688.

36. Galli J, Volante GCM, Corso ED, Almadori G, Paludetti G. Laryngeal carcinoma and laryngo-pharyngeal reflux disease. Acta Otorhinolaryngol Ital. 2006 Oct; 26(5): 260-263.

37. William WN Jr, Hong WK, Lippman SM. Chemoprevention of Cancer: Epidemiology, Prevention and Detection. In: Robert C. Bast, Jr., Carlo M. Croce, William N. Hait, Waun Ki Hong, Donald W. Kufe, Martine Piccart-Gebhart, Raphael E. Pollock, Ralph R. Weichselbaum, Hongyang Wang, James F. Holland. Holland-Frei Cancer Medicine. 9th ed. John Wiley \& Sons; 2017.

38. Califano J, Westra WH, Meininger G, Corio R, Koch WM, Sidransky D. Genetic progression and clonal relationship of recurrent premalignant head and neck lesions. Clin Cancer Res 2000 Feb;6(2):347-352.

39. Mesolella M, Iengo $M$, Testa D, Ricciardiello F, Iorio B. Chemoprevention using folic acid for dysplastic lesions of the larynx. Mol Clin Oncol 2017Nov;7(5):843-846. 